\title{
The global burden of viral hepatitis is increasing
}

Viral hepatitis has been confirmed as one of the leading causes of death and disability worldwide in a new analysis published in The Lancet. Annual deaths from viral hepatitis are now on a par with tuberculosis, HIV or malaria.

The researchers analysed data from the Global Burden of Disease study to estimate morbidity and mortality from acute infection with viral hepatitis, and cirrhosis and liver cancer caused by viral hepatitis by age group, sex and country from 1990 to 2013. Estimates were generated for acute infection with hepatitis A virus, HBV, HCV and hepatitis $E$ virus, and for cirrhosis or liver cancer secondary to HBV and HCV infection. Between 1990 and 2013, global deaths from viral hepatitis increased by $63 \%$. As a result of viral hepatitis, years of life lost, years lived with disability and disability-adjusted life-years each increased by $34 \%$ during the same time period. $\mathrm{HBV}$ and $\mathrm{HCV}$ accounted for the majority of viral-hepatitis-related mortality in 2013 (the same scenario as in 1990), with most mortality attributable to cirrhosis and liver cancer.

Interestingly, the burden of viral hepatitis varies between and within geographical regions. $\mathrm{HCV}$ infection accounts for the majority of mortality in Europe, Middle East and the Americas, whereas HBV infection accounts for the majority of mortality in sub-Saharan Africa and most of Asia. Moreover, among other leading causes of mortality, viral hepatitis is now ranked seventh, an increase in importance from previous years.

"The key now is to move from description to action," says author Graham Cooke. "We need global mechanisms to ensure access to the vaccines, diagnostics and treatment that exist to combat this challenge ... and strategic plans to bring viral hepatitis into national programming."

Katrina Ray

ORIGINAL ARTICLE Stanaway, J. D. et al. The global burden of viral hepatitis from 1990 to 2013: findings from the Global Burden of Disease Study 2013. The Lancet http://dx.doi.org/ 10.1016/S0140-6736(16)30579-7 (2016) 